\title{
The Motor Neuron Disease Mouse Model hSOD1- G93A Presents a Non-canonical Profile of Senescence Biomarkers in the Spinal Cord
}

\author{
Pascual Torres \\ IRBLleida-UdL \\ Carlos Anerillas \\ IRBLleida-UdL

\section{Mario Encinas \\ IRBLleida-UdL}

\section{Monica Povedano}

Hospital de Bellvitge

Pol Andrés-Benito

CIBERNED

Isidro Ferrer

CIBERNED

Victòria Ayala

IRBLleida-UdL

Reinald Pamplona

IRBLleida-UdL

Manuel Portero-Otin ( $\square$ manuel.portero@mex.udl.cat)

IRBLleida-UdL https://orcid.org/0000-0002-1823-0299

\section{Short report}

Keywords: Amyotrophic lateral sclerosis, navitoclax, senolytic, neuroinflammation, therapy, cell cycle, cryptic exon

Posted Date: December 23rd, 2020

DOI: https://doi.org/10.21203/rs.3.rs-104871/v2

License: (c) (i) This work is licensed under a Creative Commons Attribution 4.0 International License.

Read Full License 


\section{Abstract}

Recent evidence demonstrates a pathological role for senescent cells in Alzheimer's and Parkinson's diseases. The present study aimed to show senescence mechanisms including senescence-associated secretory phenotype (SASP) in the familial amyotrophic lateral sclerosis (ALS) transgenic mouse model hSOD1-G93A. We evaluated, as senescence biomarkers, the expression of $p 16$ and $p 21$ with reversetranscriptase quantitative PCR (RT-qPCR), immunofluorescence (IF), and immunohistochemistry (IHC), as well as the senescence-associated $\beta$ galactosidase (SA- $\beta$-gal) activity in the lumbar spinal cords (LSC) of this model. As SASP markers, we quantified the mRNA levels of $/ / 1 a$, $/ / 6$, Ifna, and Ifnb. Furthermore, we explored if an alteration of alternative splicing is associated with senescence phenomena in this model. Thus, we quantified the Adipor2 cryptic exon inclusion levels, a specific splicing variant repressed by TARDNA binding of $43 \mathrm{kDa}$ (TDP-43), using RT-qPCR. Our results show an atypical senescence-profile in LSC from transgenic mice, increasing $p 16$ and $p 21$ mRNA and protein levels in glial cells with a mostly cytoplasmic pattern, without the canonical increase in SA-beta-gal activity in these cells. Consistent with enhanced SASP, there is an increase in $/ / 1 a$ and $/ / 6$ expression. Also, TDP-43 splicing activity is compromised in this ALS model, in a direct relationship with the increase in p16 expression. However, senolytic drug Navitoclax -with reported benefits in Alzheimer and Parkinson disease mouse models does not alter the present model's disease progression. Navitoclax neither eliminates cells expressing senescence and nor represses the expression of SASP related genes. Globally, our findings support the existence of a non-canonical profile of senescence biomarkers in the LSC of the ALS model hSOD1-G93A.

\section{Introduction}

Aging is a major risk factor for developing amyotrophic lateral sclerosis (ALS) (Niccoli et al. 2017). ALS is a neurodegenerative disease characterized by the loss of motor neurons with an unfavorable outcome (< $5 \%$ survival at 5 years after diagnosis). Cellular senescence was first described by Hayflick in the 1960s as a limitation on division of normal cells in vitro (Hayflick \& Moorhead 1961). The cellular mechanisms behind this phenomenon were later described, with an important role for cell cycle inhibitors, highlighting p16-INK4a as the major contributor (Serrano et al. 1997). Another hallmark of senescent cells is the increase in $\beta$-galactosidase, commonly known as SA- $\beta$-gal which is associated with an increase in lysosomal biogenesis (Kurz et al. 2000). Cellular senescence has been described as a barrier against oncogenesis, with a tradeoff where these cells can develop a pro-inflammatory status known as SASP. This process reflects an attempt to induce tissue repair in which senescent cells, usually accumulating DNA damage, can stimulate its clearance by the immune system. Regarding neurodegenerative diseases, several groups have independently demonstrated the presence of senescent glial cells and SASP in the central nervous system (CNS).

Another process related to aging is the change in alternative splicing (AS), a conserved mechanism that increases the complexity of the proteome. TDP-43 regulates a large number of AS events in a complex way (Tollervey et al. 2011). Several evidence support the role of TDP-43 pathology in age-related neurodegenerative processes and physiological aging (McAleese et al. 2017). Most AS events regulated 
by TDP-43 involve the repression of a set of non-conserved (cryptic) exons which are abnormally incorporated into mRNA in ALS (Ling et al. 2015). In this line, we previously quantified the rate of inclusion of cryptic exons in nervous tissue from ALS donors and cellular models and found a positive correlation with age at death (Torres et al. 2018).

\section{Results And Discussion}

To clarify whether senescence-associated phenomena and TDP-43 dysfunction could be implicated in ALS, we measured the abovementioned variables in the familial ALS transgenic mouse model hSOD1G93A at different disease stages. The senescence markers $p 16$ and $p 21$, typical biomarkers of senescent cells (Coppé et al. 2010), were analyzed in LSC. Using two different technics (IHC and IF), we characterized the cellular expression pattern of $\mathrm{p} 16$. The results show that the expression of $p 16 \mathrm{mRNA}$ was progressively increased during disease evolution (Fig. 1A), whereas $p 21$ mRNA levels were only higher at the end-stage (Fig. 1B). p16 and p21 exhibited a predominantly cytoplasmic pattern (Fig. 1C), in contrast to recent results from an ALS rat model where it was mainly nuclear (Trias et al. 2019). As shown by IF, p16 positive cells were microglia (lba1 + cells) (Fig. 2A) and astroglia (GFAP + cells) (Fig. 2B). These results indicate dynamic changes in cellular senescence-associated markers and SASP related to disease evolution. $p 16$ expression is highly expressed before the symptomatology in our transgenic mice, similarly to p16 + microglia in LSC from transgenic rats (Trias et al. 2019). Both facts suggest a role for p16 in disease initiation and progression. Interestingly, senescence-associated cell cycle arrest in an early symptomatic stage (120d) is driven exclusively by $p 16$, whereas $p 21$ only increases later in this model. This may be seen as a result of the late-onset activation of p53 and the DNA damage response pathway, similar to what occurs in the senescence process in microglia (Stojiljkovic et al. 2019). In contrast to p21 (related to reversible cell cycle arrest or quiescence), the senescence process depends heavily on prolonged p16 expression. Strictly speaking, our work and most published articles on 'senescence' do not demonstrate an always irreversible cell cycle arrest. There may be divergent processes sharing common biomarkers (Sharpless \& Sherr 2015). This is the case with macrophage polarization, in which p16 expression and SA- $\beta$-gal activity are physiological, reversible, and not associated with cellular senescence (Hall et al. 2017). In this line, cytoplasmic p16 can regulate cell migration in a manner similar to cyclin D1 (Chen et al. 2013). This evidence reflects a convergent pathway of cell cycle- and senescence-associated proteins regulating cytoskeleton functions. In the case of ALS, cytoskeleton regulators like Rac1 and Cdc42 are implicated in the disease progression and neuroinflammation (D'Ambrosi et al. 2014). Thus, we hypothesized that cytoplasmic p16 could have a similar role in ALS. Like p16 cytoplasmic functions, p21 inhibits the ROCK/LIMK/Cofilin Pathway through MAPK signaling, inducing cytoskeleton remodeling (Tanaka et al. 2002)

We also analyzed another senescence canonical biomarker: SA- $\beta$-gal activity. The main cellular populations expressing SA- $\beta$-gal in ventral LSC are the motor neuron cells (Nissl + cells in the ventral horn, with a motor-neuron compatible cellular size). Neurons of other LSC locations and the vast majority of Nissl- do not show SA- $\beta$-gal activity (Fig. 1D and S1). Interestingly, SA- $\beta$-gal activity was reduced during disease progression in motor neurons and in a small fraction of Nissl- cells (compatible with glia). Our 
findings agree with previously shown data demonstrating that SA- $\beta$-gal activity in neurons is not associated with senescence, although it is increased in aging mouse brain (Piechota et al. 2016). Our results suggest that motor neurons contain more lysosomes in cell body than other cells, and that their biogenesis is compromised in this ALS mouse model. In this line, lysosomal mass deficit has already described in this model, highlighting a role of hSOD1 aggregates disturbing lysosomal biogenesis (Xie et al. 2015) and potentially explaining our results from the SA- $\beta$-gal activity assay.

Another marker commonly employed in senescence description is the increase in cytokines linked to SASP. In this case, we quantified the expression of typical SASP markers $/ / 1 a$ and $/ / 6$. We analyzed as well the expression of Ifna and Ifnb (corresponding to type-I IFN response) as they are postulated as latesenescence markers and could be helpful in determining senescence progression in the LSC of this model. The expression of Ifna was not detected in any of the analyzed samples (data not shown). We observed a different pattern of expression between //1a (Fig. 3A) and //6 (Fig. 3B). I/1a is increased in the pre-symptomatic stage and is known to be the upstream regulator of IL-6 in SASP (Orjalo et al. 2009). IL-6 is increased in cerebrospinal fluid in ALS, Alzheimer's, and Parkinson's disease (Chen et al. 2018). In contrast, Ifnb expression (Fig. $3 \mathrm{C}$ ) is not altered, which could indicate that senescence in this model does not evolve a late phase. Overall, this might reflect a complex interaction between senescence, SASP, and changes in reactive glial cells and neurodegeneration.

Regarding TDP-43 splicing function, in mice it controls the inclusion in Adipor2 mRNA (Figure S2). In line with loss of TDP-43 function in this model, cryptic exon inclusion in Adipor2 mRNA was higher in lumbar spinal cord in end-stage mice (Fig. 3D) and positively correlated with p16 expression (Fig. 3E). The present data are the first to show specific alteration regarding splicing function in this ALS model. Notably, this process is associated with an increase in the senescence marker $p 16$, and the two processes are likely to be linked in the same pathway. Of note, increased $p 16$ seem restricted to central nervous system, as sciatic nerve does not show these changes (Fig. 3F), in contrast with Adipor2 cryptic exon inclusion, which was also increased in sciatic nerve (Fig. 3G). Noteworthy, increased Adipor2 cryptic exon was associated with loss of Adipor 2 mRNA levels, suggesting increased non-sense mediated decay in both locations (Fig. 3H).

We wanted to explore the potential benefits of senolytic treatment due to the higher expression of senescence related genes in this mouse model. We performed Navitoclax treatment following the protocol described for Alzheimer's disease mouse model (Bussian et al., 2018). The treatment was initiated at 90 days old and finished at end point (Fig. 4A). We estimated the disease progression by weight loss. Navitoclax treatment did not prevent weight loss, neither prolonged survival (Fig. 4B and 4C). Finally, we quantified senescence and SASP genes in lumbar spinal cord. None of the analyzed genes showed statistically significant differences (Fig. 4D). These results suggest differences in molecular effectors between Alzheimer's and ALS.

Navitoclax is an inhibitor of antiapoptotic protein Bcl2 (Zhu et al., 2016). Senescent cells are highly dependent of different antiapoptotic members. Senolysis is achieved when this antiapoptotic protein is 
inhibited, promoting cell death (Zhu et al., 2015). Navtioclax treatment is not enough to slow the disease progression and does not extend the survival. In contrast with data in Alzheimer's and Parkinson's disease models, this treatment does not prevent the increase of senescence and SASP markers. It suggests that senescence phenotype is not driven by Bcl2 expression of stressed or aged cells in this model (Zhu et al., 2015). Further studies are warranted to determine whether senescence-linked phenomena are mechanistically involved in this fatal disease, clearing the pathway for therapeutic development.

In the case of ALS, we speculate that Navitoclax treatment is not efficient because Bcl2 is not overactivated in our G93A mouse model (Vukosavic, Dubois-Dauphin, Romero, \& Przedborski, 1999). However, Bcl-XL, a Bcl-2 family member, is overactive in astrocytes and provides pro-survival input and may mediate the activation of toxic astroglia (Lee, Kannagi, Ferrante, Kowall, \& Ryu, 2009). It suggests that an specific inhibition Bcl-XL could have greater effects on disease progression.

\section{Conclusions}

The LSC from the hSOD1-G93A mouse, a model of familial ALS, exhibits a non-canonical profile of senescence biomarkers. This profile is characterized by an early increase in $p 16$ and a late increase in $p 21$, with both displaying a mainly cytoplasmic pattern in glial cells without an increase in SA- $\beta$-gal activity. In the case of SASP, it also has a dynamic profile with increasing levels of /11a from the presymptomatic stage onward and an acute peak of expression in end-stage transgenic mice. Regarding AS, this tissue shows a dysfunctional splicing activity of TDP-43 in end-stage ALS mice. This is the first time that senescence markers, SASP, and TDP-43-associated splicing dysfunction have been described in this ALS mouse model.

\section{Methods}

\section{Experimental procedures}

\section{Animal Experiments}

A colony of the strain B6.Cg-Tg(SOD1*G93A)1Gur/J JAX catalogue stock number 004435; from now on hSOD1G93A or G93A) was purchased at The Jackson Laboratories (Bar Harbor, MN, USA). Mice were maintained in C57BL/6J background. Genotyping was performed following manufacturer's instructions. After genotyping and weaning, animals were placed at $12: 12$ hours dark / light cycle, at $22 \pm 2{ }^{\circ} \mathrm{C}$ temperature, $50 \% \pm 10$ relative humidity, in individual cages (at 21 days). For age-related studies, we evaluated. Navitoclax (T2101, Targetmol) was diluted in 60\% Phosal 50 PG (Lipoid), 30\% PEG400 (Sigma, 91893) 10\% EtOH. Navitoclax was administered by oral gavage at a dose of $50 \mathrm{mg} \mathrm{kg}-1$ body during five consecutive days followed by 16 days of rest ( $\mathrm{N}=5$ per group). Treatment cycles were repeated until clinical endpoint (righting reflex $>20 \mathrm{~s}$ ). Spinal cords and sciatic nerves were rapidly excised, frozen in liquid $\mathrm{N}_{2}$ and stored at $-80^{\circ} \mathrm{C}$. This study was approved by the Animal Research and Ethics Committee at the University of Lleida. 


\section{Cell culture}

3T3 cells were maintained in Dulbecco's Modified Eagle's Medium (11965, Thermo Fisher Scientific), 10\% FBS (10270, Thermo Fisher Scientific), $100 \mathrm{U} / \mathrm{ml}$ Penicillin-Streptomycin (15140-122, Thermo Fisher Scientific) at $37^{\circ} \mathrm{C}$ and $5 \%$ CO2. For silencing, $20 \mathrm{nM}$ (final concentration) of TARDBP siRNA mmsiTDP43s: 5'-AGGAAUCAGCGUGCAUAUA-3' mmsiTDP-43as: 5'-UAUAUGCACGCUGAUUCCU-3' (siTDP) or scrambled siRNA (siCTL) was mixed in $100 \mu$ Opti-MEM (31985062, Thermo Fisher Scientific) with $2 \mu l$ of RNAiMAX (13778100, Thermo Fisher Scientific) on the bottom of the well and incubated for 20 minutes at room temperature. $2 \mathrm{ml}$ of DMEM (11965092, Thermo Fisher Scientific) supplemented with $10 \%$ FBS containing 100000 HeLa or 3 T3 cells/well were seeded onto transfection mix. After 24 hours, transfection media was removed and changed to DMEM 10\% FBS media. 48 hours post-transfection, cells were collected for posterior analyses.

\section{Western Blot}

Protein from platelets was extracted adding $100 \mu \mathrm{L}$ of radioimmunoprecipitation (RIPA) buffer with Protease Inhibitor Cocktail (1X) to the pellet. After sonication, protein quantification was performed with Bradford assay (5000006, Bio-Rad). Fifteen $\mu \mathrm{g}$ of protein were loaded onto a $12 \%$ acrylamide SDS-PAGE gel. Membranes were blocked with I-Block (T2015, Thermo Fisher Scientific) for 1 hour and incubated overnight with primary incubated anti-TDP-43 (10782-2-AP, Proteintech) 1:1000 in TBS-T 0.05\%. After primary antibody incubation, membranes were washed 3 times with TBS-T $0.05 \%$ and incubated with secondary antibody for $1 \mathrm{~h}$. Immobilon ${ }^{\text {TM }}$ Western Chemiluminiscent HRP Substrate (WBKLS0500, Merck Millipore) was used for immunodetection. Membranes were stained with Coomassie Brilliant Blue G (27815, Sigma) for normalization. Specific bands were quantified with ImageLab v5.2.1 (Bio-Rad).

\section{RNA extraction, cDNA synthesis and RT-qPCR}

$1 \mathrm{ml}$ of TRIzol reagent (Thermo Fisher Scientific, AM9738) was added to 50-100 mg of tissue. The tissue was then mechanically homogenized using T 10 basic ULTRA-TURRAX® (IKA). $200 \mu$ l of chloroform was added to each sample and mixed. After 5 minutes of incubation at room temperature, the samples were centrifuged at $12,000 \mathrm{xg}\left(15 \mathrm{~min}, 4^{\circ} \mathrm{C}\right)$ to separate the phases. The aqueous phase was separated into a new tub and mixed by vortexing with $500 \mu \mathrm{l}$ of isopropanol. After an incubation of 10 minutes at room temperature, RNA was precipitated through spun at $12,000 \mathrm{xg}\left(10 \mathrm{~min}, 4^{\circ} \mathrm{C}\right)$. The resulting supernatant was removed, and the pellet was washed with a $75 \%$ ethanol. After vortexing, the samples were centrifuged again at $12,000 \mathrm{xg}\left(10 \mathrm{~min}, 4^{\circ} \mathrm{C}\right)$. The supernatant was discarded, and the RNA pellet was allowed to air dry at room temperature. The RNA was resuspended with $50 \mu \mathrm{l}$ of RNAse-free water, quantified with Nanodrop (Nanodrop technologies, ND-1000 UV/Vis Spectofotometer) and stored at $-80^{\circ} \mathrm{C}$ unjtil further use. One microgram of RNA was used for retrotranscription employing TaqMan Reverse Transcription Reagent using random hexamers (Thermo Scientific, N8080234). RT-qPCR experimets were performed using a CFX96 instrument (Bio-Rad, Hercules, California, USA) with SYBR select Master mix (Thermo Fisher Scientific, 4472908). Each $20 \mu \mathrm{L}$ of reaction contained $4 \mu \mathrm{L} \mathrm{cDNA}$, $10 \mu \mathrm{L}$ SYBR Select Master Mix, $0.2 \mathrm{nM}$ of forward primer and $0.2 \mathrm{nM}$ of reverse primer solutions and $4 \mu \mathrm{L}$ 
PCR grade water. RT-qPCR run protocol was as follows: $50^{\circ} \mathrm{C}$ for 2 minutes and $95^{\circ} \mathrm{C}$ for 2 minutes, with the $95^{\circ} \mathrm{C}$ for 15 seconds and $60^{\circ} \mathrm{C}$ for 1 minute steps repeated for 40 cycles; and a melting curve test from $65^{\circ} \mathrm{C}$ to $95^{\circ} \mathrm{C}$ at a $0.1^{\circ} \mathrm{C} / \mathrm{s}$ measuring rate. $\mathrm{N}=3-10$ mice per group were used for RT-PCR experiments. Primers employed in these experiments are listed in Supplementary Table S1. Actb expression was used as housekeeping to normalize the other genes. Specific Adipor2 cryptic mRNA (primers annealed with cryptic exon) was normalized with Adipor2 normal transcript (the primers annealed with conserved exons).

\section{Senescence-associated $\beta$-galactosidase activity}

Briefly, paraformaldehyde-fixed frozen sections were incubated with X-gal solution (20 mg/ml X-Gal (SIGMA), $5 \mathrm{mM} \mathrm{K}_{3} \mathrm{Fe}(\mathrm{CN})_{6}, 5 \mathrm{mM} \mathrm{K}_{4} \mathrm{Fe}(\mathrm{CN}) 6$ and $2 \mathrm{mM} \mathrm{MgCl}_{2}$ ) in PBS at pH 6.0 overnight at $37^{\circ} \mathrm{C}$. To allow comparison between specimens, all samples were assayed simultaneously. Then, neurons were stained with Green Fluorescent Nissl Stain (Thermo Fisher Scientific, N21480) diluted 1:150 in PBS and incubated 20 minutes at room temperature. The slices were then washed $3 x$ with PBS for 10 minutes at room temperature and $1 \mathrm{x}$ with PBS for 2 hours at room temperature. Nuclei were stained with DAPI (SIGMA, 32670). Images of the stained sections were taken using inverted microscope (Olympus, IX71S8F-2). Eight randomly selected areas of each mouse ( $N=2-3$ per group) of the ventral horn of lumbar spinal cord sections were photographed at 20x magnification for visual analysis. The whole section of SA- $\beta-$ Gal stained slices was photographed at 4x magnification.

\section{Immunofluorescence}

One control and one transgenic lumbar spinal cord were fixed in $4 \%$ paraformaldehyde made in PBS overnight at $4{ }^{\circ} \mathrm{C}$ and cryopreserved in 30\% sucrose in PBS 48 hours. The lumbar spinal cord was then cut at a $16 \mu \mathrm{m}$ section depth and resulting seeded on a gelatin-coated slide. Samples were permeabilized with $0.3 \%$ Triton X-100 PBS for 30 min and blocked with 5\% BSA in PBS for $1 \mathrm{~h}$ at room temperature. The primary antibody 1:100 anti-p16 (abcam, ab54210) and 1:200 anti-GFAP (abcam, ab7260) were diluted in $0.3 \%$ Triton X-100 PBS and incubated overnight at $4{ }^{\circ} \mathrm{C}$. The slices were washed with PBS three times for 5 min at room temperature, followed by the secondary antibody (diluted 1:800 in PBS), goat anti-mouse Alexa Fluor 555 (Thermo Fisher Scientific, A21422) and goat anti-rabbit Alexa Fluor 488 (Thermo Fisher Scientific, A11008) incubation for $1 \mathrm{~h}$ at room temperature in darkness. Sections were finally counterstained with $1 \mu \mathrm{g} / \mathrm{ml}$ 4,6-diamidino-2-phenylindole dihydrochloride (DAPI) in PBS for 10 min at RT and mounted on slides with Fluoromount-G® (Southern Biotech, 0100). Samples were imaged using a laser scanning confocal microscope Olympus FluoView FV10.

\section{Immunohistochemistry}

One control and one transgenic paraformaldehyd fixed paraffin embedded tissue slides were dried for $1 \mathrm{~h}$ at $65^{\circ}$ before pre-treatment procedure of deparaffinization, rehydration and epitope retrieval in the PreTreatment Module (Agilent Technologies-DAKO, PT-LINK) at $95^{\circ} \mathrm{C}$ for $20 \mathrm{~min}$ in 50x Tris/EDTA buffer, pH 9. For p21 immunohistochemical staining, p21WAF1/Cip1 antibody (Agilent Technologies-DAKO, clone SX118) 1:100 dilution was used. After incubation, the reaction was visualized with the EnVisionTM FLEX 
Detection Kit (Agilent Technologies-DAKO) using diaminobenzidine chromogen as a substrate. For p16 immunohistochemical staining, p16 INK4a antibody was used using CINtec ${ }^{\circledR}$ Histology Kit (ROCHE, clone E6H4) following manufacturer's instructions. Sections were counterstained with hematoxylin.

\section{Statistical Analysis}

All statistical tests and graphs were performed using GraphPad Prism 6 (GraphPad Software).

Normalized mRNA expression was analyzed with ordinary Two-way ANOVA test of the variables time and genotype. For multiple comparisons between genotypes, Bonferroni's multiple comparisons test was used. In SA-beta-gal activity experiments, p-value was determined by Chi-square's test. To evaluate relationship between $p 16$ and Adipor2 cryptic mRNA levels a linear regression was tested.

\section{Abbreviations}

ALS: amyotrophic lateral sclerosis

AS: Alternative splicing

CNS: Central nervous system

IF: Immunofluorescence

IHC: Immunohistochemistry

LSC: Lumbar spinal cord

RT-Qpcr: Reverse-transcriptase quantitative PCR

SASP: Senescence-associate secretory phenotype

SA- $\beta$-gal: Senescence-associated beta galactosidase

TDP-43: TAR-dna binding of $43 \mathrm{kDa}$

\section{Declarations}

Ethics approval and consent to participate

This study was approved by the Animal Research and Ethics Committee at the University of Lleida.

\section{Consent to publication}

Not applicable.

\section{Availability of data and materials}


The datasets used and/or analysed during the current study are available from the corresponding author on reasonable request.

\section{Competing interests}

The authors declare that they have no competing interests.

\section{Funding}

Grants were received from the Instituto de Salud Carlos III (PI 17-000134, PI 20-0155) to MPO, from the Generalitat de Catalunya 2017SGR696 to RP, and from the Ministerio de Ciencia, Innovación y Universidades (BFU2017-83646-P, AEl,FEDER, UE) to ME. PT is a predoctoral fellow from the Spanish Ministry of Education [FPU16/01446]. Support was also received in the form of a FUNDELA Grant, RedELA-Plataforma Investigación and the Fundació Miquel Valls (Jack Van den Hoek donation). FEDER funds are acknowledged ("A way to make Europe"). These funding bodies had no roles in the design of the study and collection, analysis, and interpretation of data and in writing the manuscript.

\section{Author's contributions}

PT, PAB and IF performed IF and IHC assays; PT, CA and ME analyzed and interpreted tissue IHC slides regarding histological scores of inflammation and SA-beta-GAL staining; MPO and RP Planned the experimental approach and performed data analyses; PT, CA and ME planned and performed RT-qPCR analyses. PT, MP and VA drafted the manuscript and analyzed immunohistochemical data. IF and MPO revised and prepared final form of the manuscript. All authors read and approved the final manuscript.

\section{Acknowledgements}

This study was supported by the Scientific and Technical Service of Immunohistochemistry, Lleida Institute for Biomedical Research, Dr. Pifarré Foundation, IRBLleida. We thank T. Yohannan for editorial assistance.

\section{References}

1. Chen X, Hu Y, Cao Z, Liu Q \& Cheng Y (2018) Cerebrospinal Fluid Inflammatory Cytokine Aberrations in Alzheimer's Disease, Parkinson's Disease and Amyotrophic Lateral Sclerosis: A Systematic Review and Meta-Analysis. Front. Immunol. 9, 2122.

2. Chen Y-W, Chu H-C, Ze-Shiang Lin, Shiah W-J, Chou C-P, Klimstra DS \& Lewis BC (2013) p16 Stimulates CDC42-dependent migration of hepatocellular carcinoma cells. PLoS One 8, e69389.

3. Coppé J-P, Desprez P-Y, Krtolica A \& Campisi J (2010) The senescence-associated secretory phenotype: the dark side of tumor suppression. Annu. Rev. Pathol. 5, 99-118.

4. D'Ambrosi N, Rossi S, Gerbino V \& Cozzolino M (2014) Rac1 at the crossroad of actin dynamics and neuroinflammation in Amyotrophic Lateral Sclerosis. Front. Cell Neurosci. 8, 279. 
5. Hall BM, Balan V, Gleiberman AS, Strom E, Krasnov P, Virtuoso LP, Rydkina E, Vujcic S, Balan K, Gitlin II, Leonova KI, Consiglio CR, Gollnick So, Chernova OB \& Gudkov AV (2017) p16(Ink4a) and senescence-associated $\beta$-galactosidase can be induced in macrophages as part of a reversible response to physiological stimuli. Aging (Albany, NY) 9, 1867-1884.

6. Hayflick L \& Moorhead PS (1961) The serial cultivation of human diploid cell strains. Exp. Cell Res. $25,585-621$.

7. Kurz DJ, Decary S, Hong Y \& Erusalimsky JD (2000) Senescence-associated (beta)-galactosidase reflects an increase in lysosomal mass during replicative ageing of human endothelial cells. J. Cell Sci. 113 ( Pt 20), 3613-3622.

8. Ling JP, Pletnikova O, Troncoso JC \& Wong PC (2015) TDP-43 repression of nonconserved cryptic exons is compromised in ALS-FTD. Science 349, 650-655.

9. McAleese KE, Walker L, Erskine D, Thomas AJ, McKeith IG \& Attems J (2017) TDP-43 pathology in Alzheimer's disease, dementia with Lewy bodies and ageing. Brain Pathol. 27, 472-479.

10. Niccoli T, Partridge L \& Isaacs AM (2017) Ageing as a risk factor for ALS/FTD. Hum. Mol. Genet. 26, R105-R113.

11. Orjalo AV, Bhaumik D, Gengler BK, Scott GK \& Campisi J (2009) Cell surface-bound IL-1alpha is an upstream regulator of the senescence-associated IL-6/IL-8 cytokine network. Proc. Natl. Acad. Sci. USA 106, 17031-17036.

12. Piechota M, Sunderland P, Wysocka A, Nalberczak M, Sliwinska MA, Radwanska K \& Sikora E (2016) Is senescence-associated $\beta$-galactosidase a marker of neuronal senescence? Oncotarget 7, 8109981109.

13. Serrano M, Lin AW, McCurrach ME, Beach D \& Lowe SW (1997) Oncogenic ras provokes premature cell senescence associated with accumulation of p53 and p16INK4a. Cell 88, 593-602.

14. Sharpless NE \& Sherr CJ (2015) Forging a signature of in vivo senescence. Nat. Rev. Cancer 15, 397408.

15. Stojiljkovic MR, Ain Q, Bondeva T, Heller R, Schmeer C \& Witte OW (2019) Phenotypic and functional differences between senescent and aged murine microglia. Neurobiol. Aging 74, 56-69.

16. Tanaka H, Yamashita T, Asada M, Mizutani S, Yoshikawa H \& Tohyama M (2002) Cytoplasmic p21(Cip1/WAF1) regulates neurite remodeling by inhibiting Rho-kinase activity. J. Cell Biol. 158, 321-329.

17. Tollervey JR, Curk T, Rogelj B, Briese M, Cereda M, Kayikci M, König J, Hortobágyi T, Nishimura AL, Zupunski V, Patani R, Chandran S, Rot G, Zupan B, Shaw CE \& Ule J (2011) Characterizing the RNA targets and position-dependent splicing regulation by TDP-43. Nat. Neurosci. 14, 452-458.

18. Torres P, Ramírez-Núñez O, Romero-Guevara R, Barés G, Granado-Serrano AB, Ayala V, Boada J, Fontdevila L, Povedano M, Sanchís D, Pamplona R, Ferrer I \& Portero-Otín M (2018) Cryptic exon splicing function of TARDBP interacts with autophagy in nervous tissue. Autophagy 14, 1398-1403.

19. Trias E, Beilby PR, Kovacs M, Ibarburu S, Varela V, Barreto-Núñez R, Bradford SC, Beckman JS \& Barbeito $L$ (2019) Emergence of microglia bearing senescence markers during paralysis progression 
in a rat model of inherited ALS. Front. Aging Neurosci. 11, 42.

20. Xie Y, Zhou B, Lin M-Y \& Sheng Z-H (2015) Progressive endolysosomal deficits impair autophagic clearance beginning at early asymptomatic stages in fALS mice. Autophagy 11, 1934-1936.

\section{Table}

Table 1

SYBR Green probes used in RT-qPCR analysis.

\begin{tabular}{|ll|}
\hline Gene & Sequence \\
\hline Actb Fwd & GTGACGTTGACATCCGTAAAGA \\
\hline Actb Rev & GCCGGACTCATCGTACTCC \\
\hline p16 Fwd & CCCAACGCCCCGAACT \\
\hline p16 Rev & GCAGAAGAGCTGCTACGTGAA \\
\hline l1a Fwd & AGCGCTCAAGGAGAAGACC \\
\hline l1a Rev & CCAGAAGAAAATGAGGTCGG \\
\hline II6 Fwd & ACCAGAGGAAATTTTCAATAGGC \\
\hline II6 Rev & TGATGCACTTGCAGAAAACA \\
\hline Ifna Fwd & ATGGCTAGGCTCAGCACTTTC \\
\hline Ifna Rev & CTCACTCAGACTTGCCAGCA \\
\hline Ifnb Fwd & AGCTCCAAGAAAGGACGAACA \\
\hline Ifnb Rev & GCCCTGTAGGTGAGGTTGAT \\
\hline Adipor2 Fwd & TGTTTGTAAGGTGTGGGAAGG \\
\hline Adipor2 Rev & GTTGCCCGTCTCTGTGTGTAT \\
\hline Adipor2 cryptic Fwd & AGAAGTGGAGTTACAATTGTG \\
\hline Adipor2 cryptic Rev & AAACAAACTCTTCCATTCGTT \\
\hline p21 Fwd & TTGCCAGCAGAATAAAAGGTG \\
\hline p21 Rev & TTTGCTCCTGTGCGGAAC \\
\hline
\end{tabular}

\section{Figures}


Figure 1
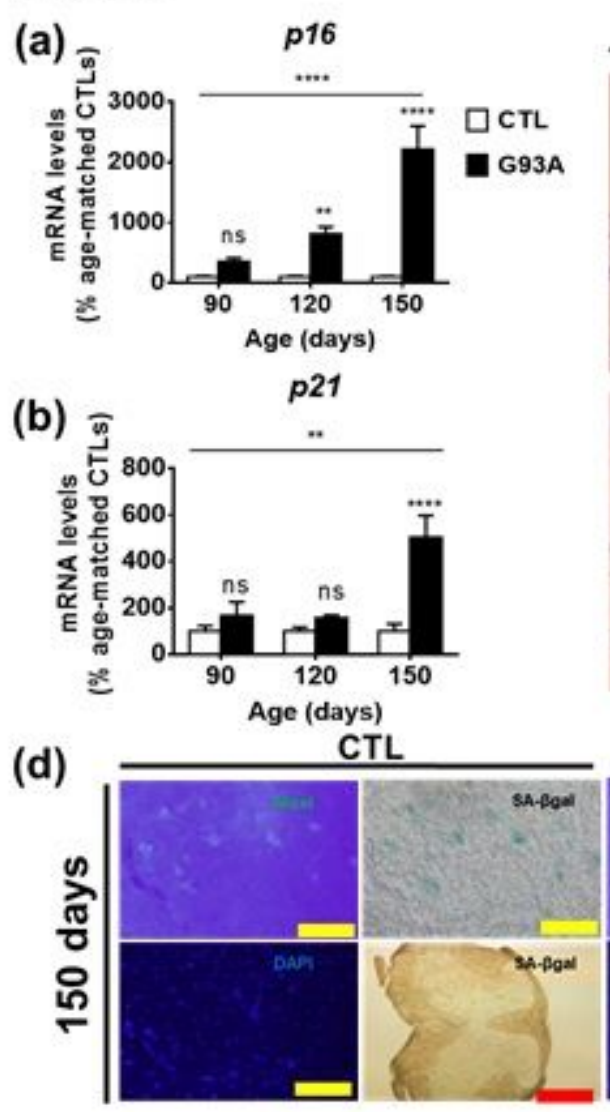
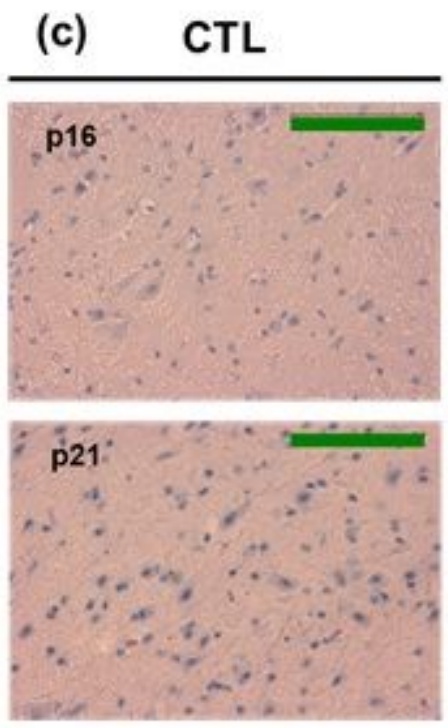

hSOD1-G93A

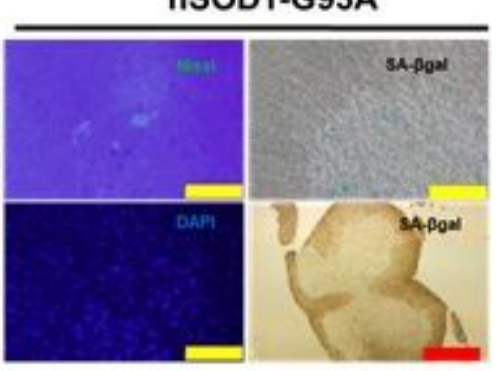

\section{hSOD1-G93A}
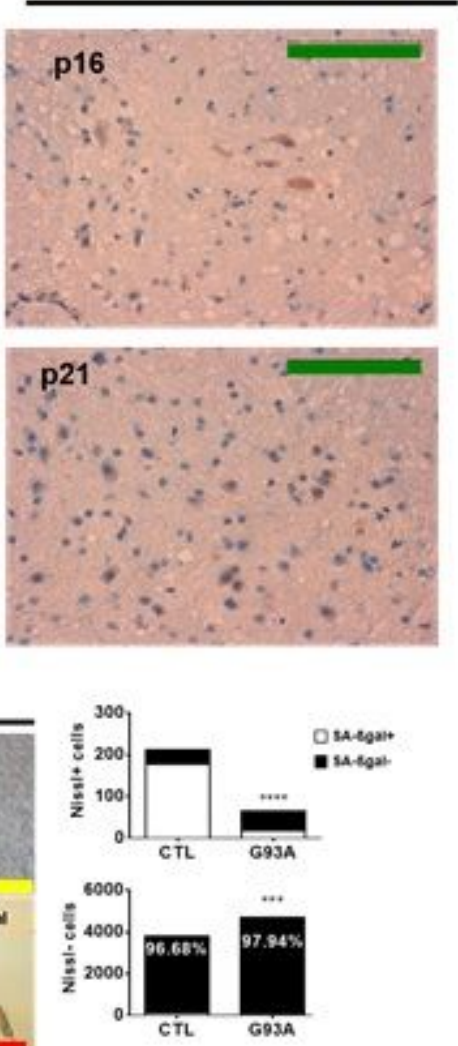

\section{Figure 1}

Increase in senescence markers in spinal cord during ALS progression. p16 expression was progressively higher at 120 days and 150 days (a), whereas p21 were only found to be increased at end stage (b). Both p16 and p21 exhibited cytoplasmic staining (c). Nissl+ cells of the ventral horn of the spinal cord (compatible with motor neurons) are the main contributor to SA-beta-gal activity in lumbar spinal cord and are almost depleted in this activity in hSOD1-G93A mice at 150 days, similarly to Nissl- (glia) cells (d). p16 and p21 expression are expressed as mean \pm SEM. Ns indicates $p>0.05$; $* \star p<0.01 ; * \star * p<0.001$; $\star \star \star \star p<0.0001$ for Student's $t$ test or ANOVA, when appropriate. Red arrows indicate $p 16$ and p21 positive cells. Green scale bar represents $100 \mu \mathrm{m}$, yellow scale bar represents $500 \mu \mathrm{m}$, and red scale bar represents $2500 \mu \mathrm{m}$. $\mathrm{n}=4$ from each genotype and age. 


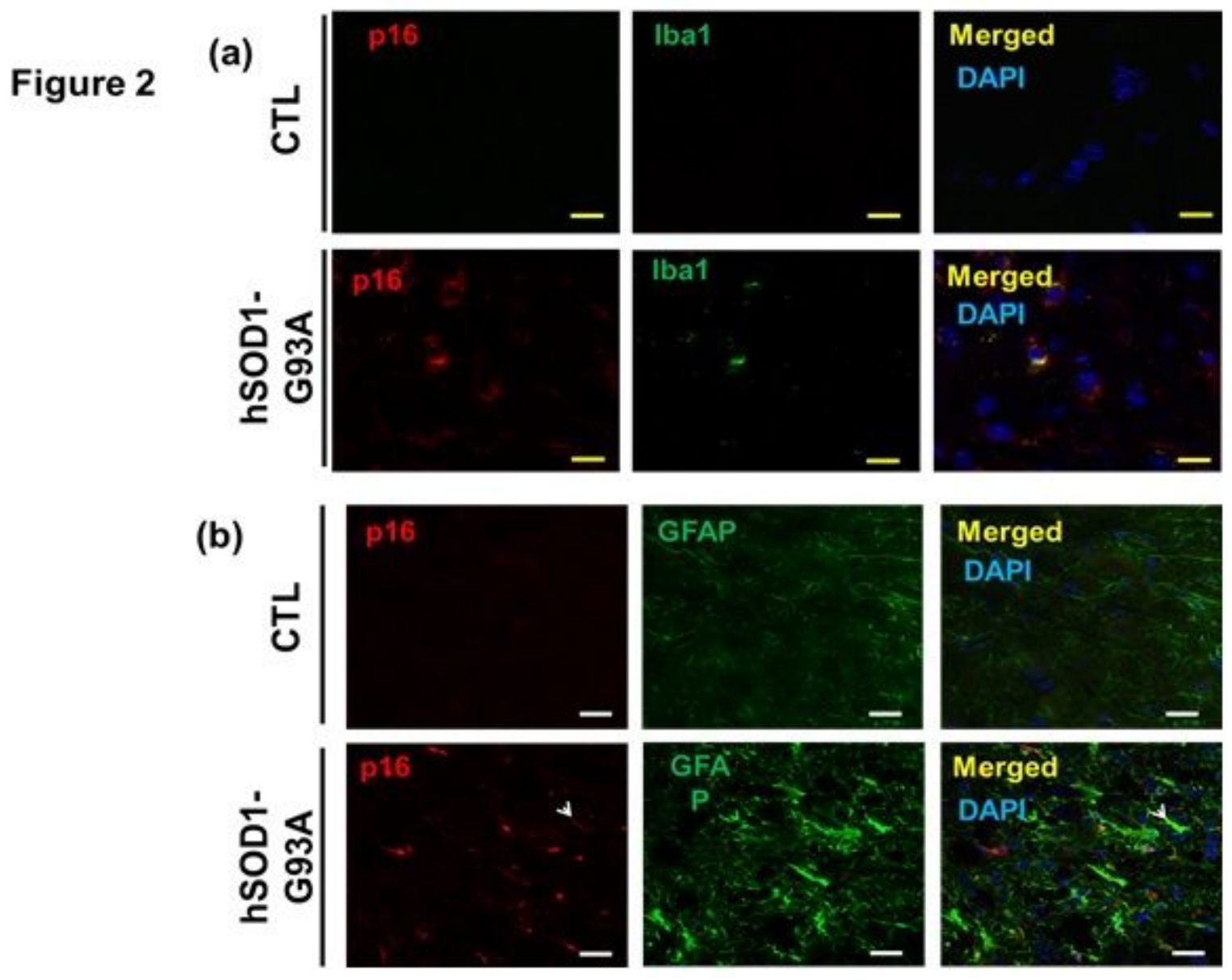

Figure 2

Cytoplasmic p16+ microglia (Iba1+) (a) and astrocytes (GFAP+) (b) are present in lumbar spinal cord of transgenic mice and absent in controls. Scale bar represents $500 \mu \mathrm{m}$. Representative images from independent experiments ( $n=10$ slices from at least 3 different animals from each genotype and age) are shown. 
Figure 3
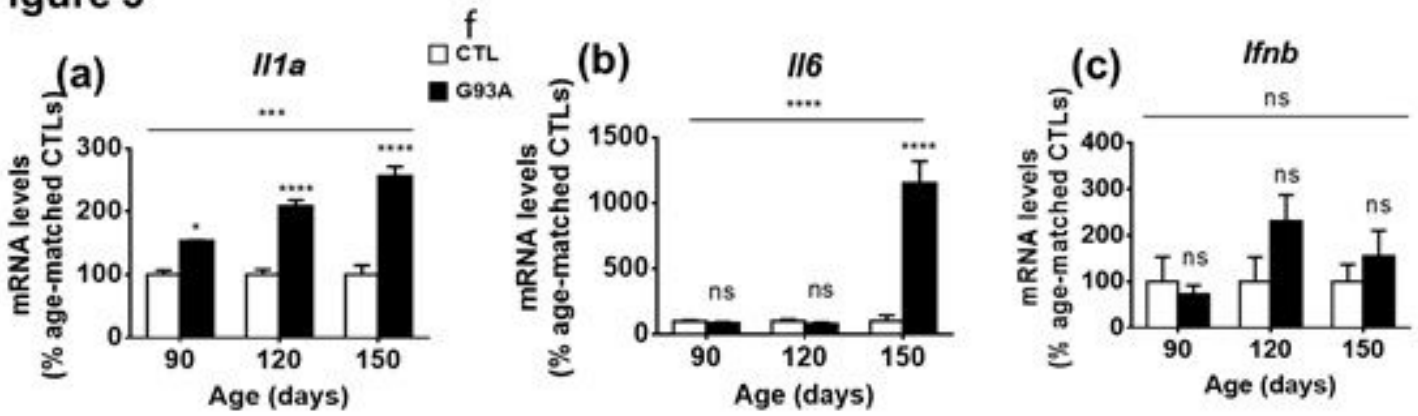

(d)

(e)
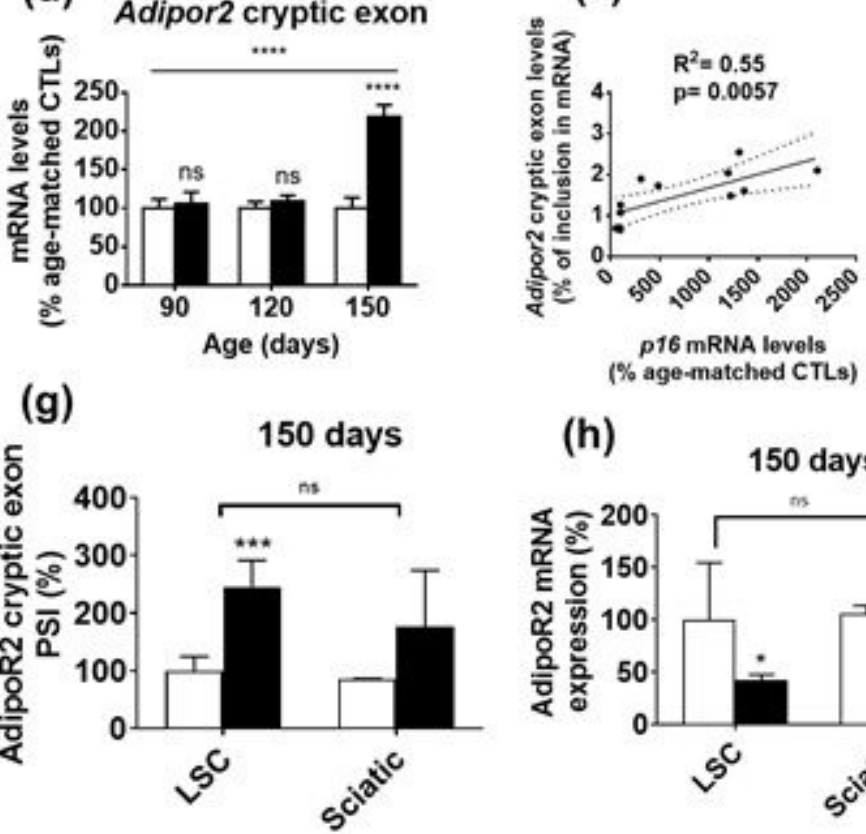

(h)

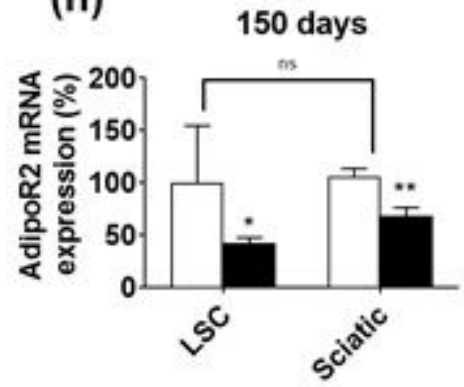

(f)

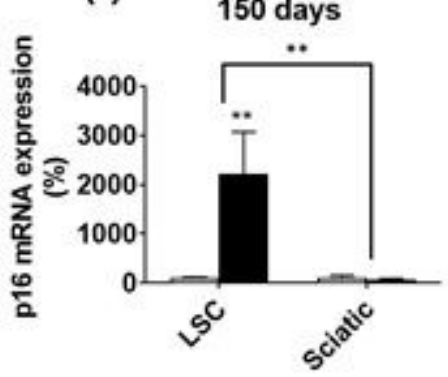

Figure 3

SASP markers and TDP-43 dysfunction are increased in LSC, but not sciatic nerve, from transgenic hSODG93A mice. II1 a mRNA was higher from pre-symptomatic stage onward in transgenic mice and increased in later stages (a). II6 expression (b) was induced in end-stage hSOD1-G93A mice, in contrast with Ifnb (c). Cryptic exon in Adipor2 mRNA was more incorporated in 150-day-old transgenic mice (d). The inclusion ratio of Adipor2 cryptic exon positively correlated with p16 expression (e) in LSC. Despite lack of increased p16 expression in sciatic nerve (f), similarly to LSC, cryptic exon in Adipor2 mRNA was more incorporated in sciatic nerves from 150-day-old transgenic mice (g), in association with diminished total Adipor2 mRNA in both locations (h). $\| 1 \mathrm{a}$, Il6, Ifnb, and cryptic Adipor2 expression are expressed as mean \pm SEM. $n s p>0.05 ;{ }^{\star} p<0.05 ;{ }^{\star \star *} p<0.001 ;{ }^{* \star \star \star} p<0.0001$. $n=4$ from each genotype and age. 


\section{Figure 4}

(a)

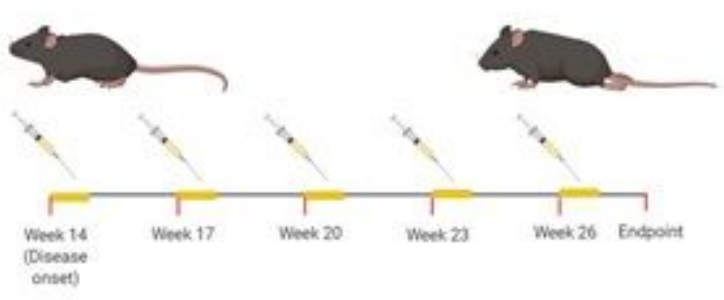

(c)

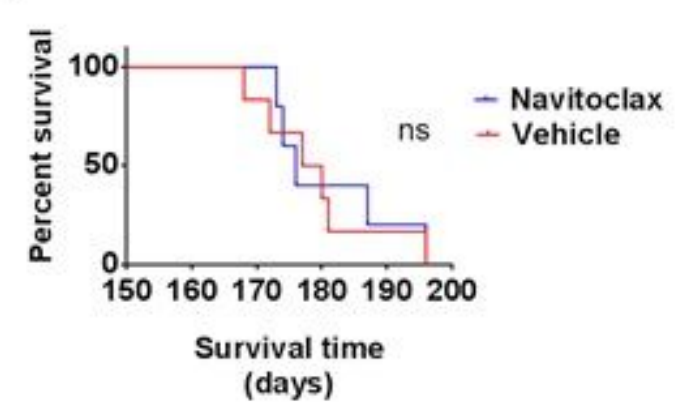

(b)

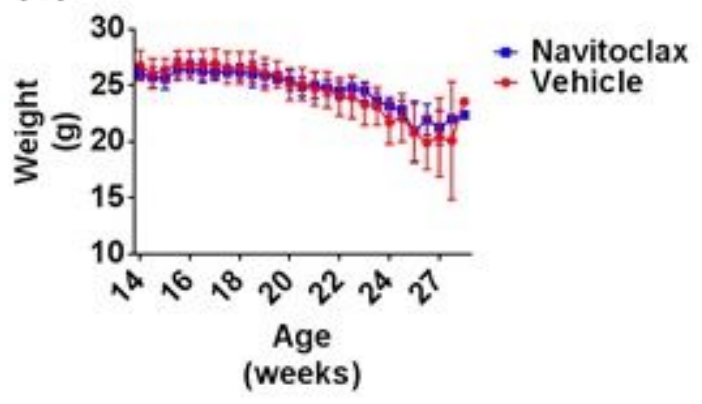

(d)

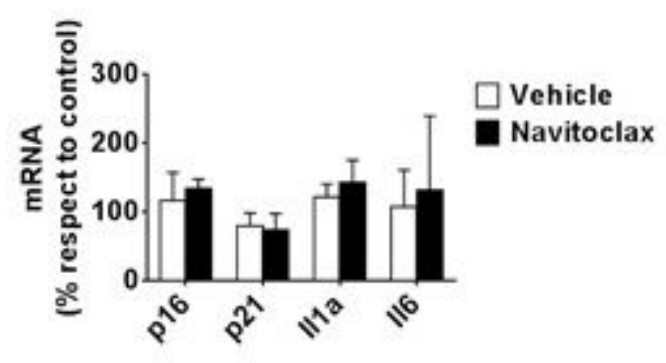

\section{Figure 4}

Senolytic treatment using Navitoclax does not slow the disease progression. A chronic treatment was established with five consecutive doses followed by two weeks of resting (a). Weight loss was not different between Navitoclax and vehicle experimental groups (b). Survival time was also unaltered between groups (c), as senescence and SASP genes (d). $n=5$ from each genotype.

\section{Supplementary Files}

This is a list of supplementary files associated with this preprint. Click to download.

- FigS1.jpg

- FigS2.jpg 